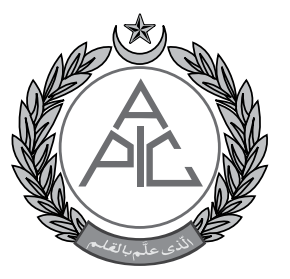

\title{
Continuous electroencephalography (cEEG) monitoring in critically ill patients
}

\author{
Azham Purwandhono ${ }^{\mathrm{a}, \mathrm{b}}$, Abdulloh Machin ${ }^{\mathrm{a}}$, \\ Wardah Rahmatul Islamiyah ${ }^{a}$, Hanik Badriyah Hidayati ${ }^{\mathrm{a}}$
}

\begin{abstract}
Critically ill patients need to be treated quickly, carefully, precisely and comprehensively. Neurological status should be concerned because of the severe impact that occurs if it is not immediately handled, that is permanent disability or death. The neurologic problem like non convulsive seizures may not distinctly clear. It is difficult to assess non-convulsive seizure only by clinical physical examination. Continuous Electroencephalography (cEEG) is a useful to measure electrical brain activity. It could be used to identify seizure, cerebral ischemic, monitor and evaluate the medication, and prognostic of the diseases. This equipment is noninvasive and could give real-time information about the patient condition. The complex procedure and availability of skilled technician and competent reviewer, is the barrier to optimize this tools. Besides the benefit and challenge, cEEG is recommended to be used in critically ill patients' management.
\end{abstract}

aDepartement of Neurology, Medical Faculty of Airlangga University / Soetomo General Hospital Surabaya, East Java, Indonesia

${ }^{\mathrm{b}}$ Faculty of Medicine, University of Jember, Jember, East Java, Indonesia

Correspondence:

Hanik Badriyah Hidayati, Departement of Neurology, Medical Faculty of Airlangga University / Soetomo General Hospital Surabaya, East Java, Indonesia; E-mail: hanikhidayati@yahoo.com

Received: 19 Oct 2018

Reviewed: 28 Oct 2018

Accepted: 5 Nov 2018

Key words: $c E E G$, seizure, non-convulsive, critically ill

Abbreviations used: ADR - Alpha to Delta Ratio; CBF - Cerebral Blood Flow; CEEG continuous Electroencephalography; CNS - Central Nervous System; CT - Computed Tomography; DCI - Delayed Cerebral Ischemia; EEG - electroencephalography; GCS - Glasgow Coma Scale; ICU - Intensive Care Unit; MRI - Magnetic Resonance Imaging; NCS - Non-Convulsive Seizures; NCSE - Non-Convulsive Status Epilepticus; PAV Percent Alpha Variability; PEDs - Periodic Epileptiform Discharges; RAV - Relative Alpha Variability; TBI - Traumatic Brain Injury; TCD - Transcranial Doppler

Citation: Purwandhono A, Hidayati HB, Machin A, Islamiyah WR. Continuous electroencephalography (cEEG) monitoring in critically ill patients. Anaesth Pain \& Intensive Care 2018;22 Suppl 1:S46-S52

\section{INTRODUCTION}

Critically ill patients have a high risk of neurologic problems. If this condition is not realized and not detected early, it will cause morbidity and mortality outcomes. The main goal in the management of critically ill patients is to identify, prevent, and treat neurologic/underlying disease that may cause secondary brain injury. ${ }^{1}$ Neurologic monitoring is important. Electroencephalography (EEG) is a tool to detect, monitor, and evaluate brain activity. This is a noninvasive tool to assess brain function and may give useful data in the diagnosis and prognosis of cerebral complications. ${ }^{2,3}$ Continuous EEG monitoring
(cEEG) provides an important real-time information in detecting subclinical seizures and non-convulsive status epilepticus in the critically ill patients. Therefore, it may be assumed to be the gold standard for seizure detection. ${ }^{4,5}$ This review will emphasize the indication, method, benefit, and challenge of cEEG. The English publication searching method was using PubMed and Google Scholar database. The search term included continuous EEG, critically ill, non-convulsive seizure.

\section{INDICATIONS}

In Intensive Care Unit (ICU), EEG is performed 
on patient with decreased level of consciousness with unknown cause and indicated to detect nonconvulsive seizure, ischemia, to monitor sedation and to assess prognosis after cardiac arrest, acute brain injury, hypoxic-ischemic encephalopathy, acute ischemic stroke, intracerebral hemorrhage, subarachnoid hemorrhage, infectious and noninfectious encephalitis, severe sepsis and supporting data to diagnose electrocerebral inactivity caused by brain death. ${ }^{6-8}$

\section{Detection of Non-convulsive Seizure:}

cEEG is indicated for unconscious patients with unclear cause. This examination is performed from 24 to 48 hours for patient with unknown neurologic injury. ${ }^{9}$ In critically ill patient, seizure is dominated by non-convulsive seizures (NCS) or non-convulsive status epilepticus (NCSE) and EEG monitoring is needed for detection. There are many causes of NCSE, such as stroke, head trauma, CNS infection, sepsis, and organ transplantation. ${ }^{10,11}$

NCS is seizure without prominent motor symptom. It has a subtle clinical presentation, such as agitation, catatonia, psychosis, blinking, staring, nystagmus, facial muscle/limb twitching, and associated with altered consciousness. The duration is at least 10 seconds. NCSE is continuous seizure or intermittent electrographic seizure without prominent motor symptom for minimum 30 minutes in comatose patient and without recovery between episodes. There were no definitive diagnostic criteria for NCSE identification, but there were many criteria proposed by Chong, Sutter, and Salzburg. ${ }^{12}$ NCS and NCSE were found in $8-48 \%$ of comatose patients. ${ }^{12-14}$ The probability of seizure detection during cEEG increases with the duration of monitoring. Claasen et al, (2004) reported that seizures occurred in 110 (19\%) of 570 patients (1996-2002) who underwent cEEG monitoring in Columbia University New York - Presbyterian Hospital. Most seizures was nonconvulsive (92\%). It was confirmed that subclinical seizures were extremely common in these patients. The first seizure was recorded after 24 hours in $20 \%$ of comatose patients. Only $56 \%$ of patients had their first event within 1 hour of EEG, and this percentage increased to 82,88 , and $93 \%$ within 12,24 and 48 hours, respectively. ${ }^{15}$

If seizure is not detected and stopped, it can cause permanent brain damage and death. ${ }^{5}$ Seizure can increase metabolic demand and blood flow, worsen secondary brain injury, increase in tracranial pressure, worsen midline shift, alter tissue oxygenation, decrease hippocampal mass and cause neuronal necrosis. ${ }^{16,17}$ Delay in the detection and management of seizure may worsen the condition and could increase morbidity and mortality rate. ${ }^{6}$ Patients with NCSE were associated with prolonged ICU stay and poor outcomes with $20 \%$ to $30 \%$ mortality rate. However, this poor outcome is strongly influenced by the underlying etiology. ${ }^{3}$

It was found electrographic seizures in $29 \%$ of patients with acute and severe metabolic disorder. There are many metabolic derangements that provoke seizure, such as hypoglycemia, hyperglycemia, hyponatremia, hypocalcemia, hypercalcemia, endocrine, hepatic and renal dysfunction. EEG pattern give a little specific information about source of metabolic derangements, but some pattern could give typical metabolic source, like triphasic waves frequently seen in hepatic and renal dysfunction. Study from Cipto Mangunkusumo Hospital Jakarta reported incidence NCSE is high (61.8\%) in metabolic encephalopathy based on Salzburg criteria. The EEG patterns showed epileptiform and non-epileptiform pattern, like focal slowing, general slowing, triphasic wave, and burst suppression. The treatment is antiseizure and correcting the underlying metabolic derangements. It should be immediately identified and treated before the brain injury becomes permanent. ${ }^{12,21-26}$

Traumatic Brain Injury (TBI) with depressed skull fracture, penetrating injury and large cortical contusion/hematoma is high risk for non-convulsive seizure. It is advisable in patient with Glasgow Coma Scale (GCS) below 9 or fluctuating mental status doing cEEG monitoring within 24-48 hours of admission. Seizure could happen within the first week after TBI (incidence 4-14\%). Some studies show that seizure and low Percent Alpha Variability (PAV) are independent risk factors for poor outcomes in TBI patients. $., 27,28$

Mild TBI show atypical EEG pattern, like slowing of the posterior dominant rhythm and increased diffuse theta slowing. This condition may return to normal within hours to weeks. Some condition should be considered in TBI, such as a breach artifacts in patient who did craniotomy, EEG attenuation pattern in subgaleal hematoma patient. Epileptiform abnormalities such as sporadic spikes and sharp waves, periodic epileptiform discharges, and rhythmic patterns are at high risk for post traumatic epilepsy. ${ }^{29-32}$

\section{Detection of Cerebral Ischemia:}

Cerebral ischemia is found in approximately 30\% patient with vasospasm and aneurysmal subarachnoid 
hemorrhage. ${ }^{17}$ cEEG may detect decreasing cerebral perfusion in vasospasm. Cerebral ischemia is a risk factor for seizure. The study by Claasen et al, (2004) reported that $11 \%$ patient with ischemic stroke had seizure (mostly non-convulsive) and associated increased mortality rate. Another condition that can cause cerebral ischemia is surgical procedure, e.g. carotid endarterectomy (CEA) and intracranial hypertension. . $^{27}$

Based on Cerebral Blood Flow (CBF), there were several EEG changes correspond to cerebral ischemia. Loss of beta activity could be found at the CBF of $25-35 \mathrm{ml} / 100 \mathrm{~g} / \mathrm{min}$. If the CBF diminished of 18 $25 \mathrm{ml} / 100 \mathrm{~g} / \mathrm{min}$, it will show the theta slowing. The EEG pattern showing delta slowing at the CBF of $12-18 \mathrm{ml} / 100 \mathrm{~g} / \mathrm{min}$. The irreversible state of cerebral ischemia established at the CBF of $<8-10 \mathrm{ml} / 100$ $\mathrm{g} / \mathrm{min}$ and from this EEG pattern suggest there is suppression. ${ }^{33}$

The EEG record help to identify reduced CBF in critically ill patient who high risk of cerebral ischemia and limited information from clinical examination. It is very sensitive to ischemia and may reveal changes at a reversible stage of reduced $\mathrm{CBF}$ and neuronal dysfunction $(25$ to $30 \mathrm{~mL} / 100 \mathrm{~g} / \mathrm{min}){ }^{9,27}$ The more rapidly decrease $\mathrm{CBF}$, the more severe change could be seen in EEG. EEG alteration happens within seconds of decrease CBF. Vasospasm could be immediately detected in EEG, and the intervention could be soon applied. MRI and CT Scan can also detect cerebral ischemia but it needs time to be seen in imaging, usually infarction has occurred and the intervention seems to be late. The cEEG pattern that related to vasospasm consist of focal delta slowing associate with the injury area, burst of frontal biphasic delta waves, continuous rhythmic delta activity, continuous rhythmic delta activity, and continuous polymorphic or unreactive delta. ${ }^{2,12,17}$

Delayed Cerebral Ischemia (DCI) is a severe complication caused by vasospasm and/or subarachnoid hemorrhage. Suspicious diagnosis is made from clinical presentation, such as decrease of consciousness, new neurological deficit. But in comatose patient, the new neurological deficit presentation is difficult to evaluate. Angiography could identify DCI during vasospasm but it is invasive procedure and need the patient to move to radiology room. Transcranial Doppler (TCD) could be used to identify ischemia by measure cerebrovascular velocity in only short time. Thus, cEEG is recommended noninvasive tools to identify DCI during vasospasm in long period time. The limitation of cEEG is difficulty in ischemia identification at subcortical region because of the position of electrode on the scalp, far away to detect electrical brain activity. 2,17,27

Quantitative EEG could help cEEG for detection DCI. Alpha to Delta Ratio (ADR) and Relative Alpha Variability (RAV) are the useful parameter to detect DCI and correlate with the outcome of SAH. The decrease of ADR and RAV (>38\%) indicate DCI and it could be detected in 7 hours before clinical deterioration and 44 hours before the imaging (CT scan) show ischemic lesions. The recent study proposes cEEG monitoring in all aSAH patients. Early detection and intervention of DCI may improve outcome. . $^{912,34}$

\section{Monitoring and Evaluating Therapy:}

cEEG is recommended to monitor and evaluate seizure, either convulsive and non-convulsive seizure. The improvement of seizure could be seen from the EEG pattern and mental status which achieved from bedside examination or video recording. After seizure have been controlled and the medication stopped, cEEG still continue for at least 24 hours because of the high risk seizure recurrence, especially in comatose patient and/or have Periodic Epileptiform Discharges (PEDs). ${ }^{13,35}$

Treating the seizure to achieve burst suppression in EEG is effective in preventing breakthrough seizure. It could be severe clinical presentation when this seizure happen. Jordan and Hirsch (2006) reported the frequency of breakthrough seizures is low (4\%) when burst suppression achieved, conversely the frequency is high 53\% if the treatment just only eliminates EEG epileptiform activity (seizure suppression). Cerebral metabolic rate and cerebral blood volume decreases in burst suppression so it can provide patient's brain protection. ${ }^{9,36}$

Sedation is used in $42 \%$ to $72 \%$ of critically ill patients in order to make patient comfort and safe during application of mechanical ventilator. There are many scales have been developed to monitor sedation administration, such as Ramsay Scale, Riker Sedation Agitation Score (SAS) and the Richmond AgitationSedation Scale (RASS). cEEG is considered to assess the degree of sedation when the patient is unresponsive and the scales cannot be used. Burst suppression which found in cEEG is not the only indicator for monitoring NCS or NCSE but also for monitoring of sedation administration. Burst suppression help to titrate the optimal dose of sedation with minimal adverse effects, such as hemodynamic complication, cardiac distress renal and hepatic failure. The 
maintenance of burst suppression may improve the survival. The burst suppression in patient who have pentobarbital titration had a good recovery (24\%) with minimal or no disability $(10 \%)$ at 1 year. ${ }^{9,27,35,37}$

\section{Prognostication of Disease:}

The data collected from cEEG help to predicting the outcome of comatose patients caused by several neurologic conditions, like TBI, subarachnoid hemorrhage, hypoxic encephalopathy after cardiac arrest. The presence of seizure (found in $18 \%$ of patients with TBI) and the absence of normal sleep architecture will give poor outcome because of the occurrence of secondary brain injury and metabolic distress. The other study by Vespa et al. (2002) reported that reduced percentage of alpha variability in patient with moderate or severe TBI within 3 days after injury is highly predictive for poor outcome. $9,27,28$

In $\mathrm{SAH}$, cEEG is an independent factor to predict the outcome. The patient had good outcome if it is obtained physiologic sleep architecture, state changes, reactivity to external stimuli, and absence of periodic discharges. Conversely, Epileptiform discharges, NCSE and absent EEG background reactivity associated with poor outcome (modified Rankin Scale 4 to 6). The mortality is high (30\%$100 \%$ ) when the NCSE was recorded and prolonged. The survivor will still dependent. ${ }^{28,35}$

The treatment of cardiac arrest is hypothermia and sedation administration. The clinician should be aware that seizure (subclinical) could happen after this treatment discontinue. The cEEG is required to identify seizure because most of it is non-convulsive. The presence of burst-suppression or status epilepticus and the absence of EEG reactivity to external stimuli at 3 days after cardiac arrest significantly predict poor outcome. , $^{2,35}$

The most important thing to realize the EEG pattern, that sometimes give a false positive in the outcome prediction. The EEG shows an improvement but still poor of the clinical outcome. ${ }^{35}$

\section{INSTRUMENTATION}

Ideally, cEEG should be available 24 hours a day and 7 days a week. This examination should be done immediately (less than 1 hour) after indication criteria are established. The duration of cEEG varies, depends on the patient characteristics. Commonly seizures are recorded during the first 24 hours, and frequently in the first 48 hours or more. ${ }^{9}$ Most NCSE (80-95\%) may be found in the first 24-48 hours of EEG recording. The obtained data from
EEG were checked and reported at least once daily by a competent clinical reviewer. ${ }^{6}$ The quality of the recorded EEG should be checked twice daily to make sure that the electrodes were placed correctly and maintained in low impedance (refilled gel). A good teamwork between EEG reviewer, technician and ICU personnel is required for the best cEEG performance. ${ }^{35}$

\section{BENEFITS}

EEG examination is a non-invasive tool to detect seizures phenomena such as subclinical seizures and non-convulsive status epilepticus. It allows "realtime" exploration of the spontaneous activity of the neurons in the cerebral cortex. ${ }^{3}$ cEEG pattern can help to detect and monitor seizures, non-convulsive seizures, ICP changes, and cerebral ischemia, to assist in titrating therapies such as barbiturate administration and to predict the outcome of comatose patients. The other tools are less suitable to detect brain activity and its dysfunction, like Magnetic Resonance Imaging (MRI) or Computed Tomography Scan (CT-Scan). Those devices can show specific anatomy imaging but cannot find out the function and pathological process in the brain. Moreover, the risk of transporting critically ill patient to radiology room is quite high. ${ }^{17,38}$

\section{CHALLENGES}

The limitations of cEEG are the high cost, the need for a skilled technician and a competent data reviewer, the technical difficulty of implementation and the susceptibility to drug effects and superficial artifacts such as those caused by eye and limb movements or electrical interference. ${ }^{1,20,39}$ Artifacts may be barrier in interpreting EEG. They may mimic seizure and could arise from chest percussion, rhythmic movement, intravenous device, ventilator tubing, dialysis and other equipment that occupies space and produces a lot of electrical noise. The EEG ground plays a role in preventing power line noise from interfering with the electrical signals from the brain. Poor grounding will produce $50 / 60 \mathrm{~Hz}$ artifact. The other obstacles while undertaking long term recordings are the chance of detachment of electrode contact that may be caused by drying out, impedance mismatches, head anatomy change, open wounds, and body movement. ${ }^{9,40-42}$

There are three types of electrodes, i.e. cup electrodes, stick-on electrocardiogram electrodes, and needle electrodes. Each type has their own strength and weakness. Cup electrode has a high sensitivity (better signal quality) and familiar for daily activity but this electrode had several limitations. It has to be 
removed for brain imaging, has a short lifespan (6-12 hours), unpleasant smell from the collodion, and risk of fire. Stick on electrode has advantages such as good adhesive properties and longer lifespan (24 hours); but this electrode also needs to be removed when imaging is needed. A higher impedance can decrease the signal quality. Needle electrode is quite invasive and expensive, but it produces the best records with the ability of long-term application and doesn't need to remove while doing brain imaging. ${ }^{40}$

In developing countries like Indonesia, which lack of skilled technicians and competent reviewers to perform cEEG. The disparity of health care, especially intensive care unit and human resource concentrate in capital cities are need to be our concern. Intensive training program and certification regularly performed by organization and network establishing have been done to overcome this challenges. The health policy from the government is also required to support and distribute these sophisticated facilities. The final purpose is to give the best medical service and improving the quality of life. ${ }^{43,44}$

\section{SUMMARY}

In critically ill patients, cEEG can provide significant information about the current neurological state, like non-convulsive seizure, cerebral ischemia, efficacy of medication and prognosis for the outcome. This examination should be combined with clinical information and physical examination to give the best treatment for patients. The challenges in procedure and availability of human resource should not hamper the application of cEEG to support the management of critically ill patients.

Conflict of interest: None. No funding was used for the preparation of this review.

\section{REFERENCES}

1. Peacock SH, Tomlinson AD. Multimodal neuromonitoring in neurocritical care. AACN Adv Crit Care. 2018;29(2):183-194. [PubMed] doi:10.4037/aacnacc2018632

2. Caricato A, Melchionda I, Antonelli M. Continuous electroencephalography monitoring in adults in the intensive care unit. Crit Care. 2018;22(1). [PubMed] [Free full text] doi:10.1186/ s13054-018-1997-x

3. Azabou E, Fischer C, Guerit JM, Annane D, Mauguiere $F$, Lofaso $F$, et al. Neurophysiological assessment of brain dysfunction in critically ill patients: an update. Neurol Sci. 2017;38(5):715-726. [PubMed] doi:10.1007/s10072-017-2824-x

4. Rossetti A0, Urbano LA, Delodder F, Kaplan PW, Oddo M. Prognostic value of continuous EEG monitoring during therapeutic hypothermia after cardiac arrest. Crit Care. 2010;14(5). [PubMed] [Free full text] doi:10.1186/ cc9276

5. Moreira JB, Veiga De Sá AL, Da Silva Campos MAFT. The colour density spectral array in the perioperative management of urgent craniectomy: Can we identify epileptiform activity? Anaesthesia, Pain Intensive Care. 2017;21(1):79-86.
6. Khawaja AM, Wang G, Cutter GR, Szaflarski JP. Continuous electroencephalography (ceeg) monitoring and outcomes of critically ill patients. Med Sci Monit. 2017;23:649-658. [PubMed] [Free full text] doi:10.12659/MSM.900826

7. Sinha SR, Sullivan L, Sabau D, SanJuan D, Dombrowski KE, Halford JJ, et al. American clinical neurophysiology society guideline 1: minimum technical requirements for performing clinical electroencephalography. J Clin Neurophysiol. 2016;33(4):303307. [PubMed] doi:10.1097/ WNP.0000000000000308

8. Banu SH. EEG in ICU: A monitoring tool for critically ill patient. Bangladesh Crit Care J. 2014;2(1):28-34.

9. Gilmore EJ, Claassen J. Continuous electroencephalogram monitoring in the ICU. In: Lee K, ed. The NeurolCU Book. 2nd ed. New York: McGraw-Hill Education; 2018:262-286.

10. Kubota $\mathrm{Y}$, Nakamoto $\mathrm{H}$, Egawa S, Kawamata T. Continuous EEG monitoring in the ICU. J Intensive Care. 2018;6(39):1-8. [PubMed] [Free full text] doi:10.1186/s40560018-0310-z

11. Murthy JMK, Naryanan TJ. Continuous EEG monitoring in the evaluation of non-convulsive seizures and status epilepticus. Neurol India. 2004;52(4):430-435. [PubMed]

12. Husain AM, Sinha SR. Continuous EEG Monitoring Principle and Practice. Switzerand: Springer International Publishing; 2017. doi:10.1007/9783-319-31230-9

13. Abend NS, Dlugos DJ, Hahn CD, Hirsch LJ, Herman ST. Use of EEG monitoring and management of nonconvulsive seizures in critically ill patients: A survey of neurologists. Neurocrit Care. 2010;12(3):382-389. [PubMed] [Free full text] doi:10.1007/ s12028-010-9337-2

14. Schmitt SE. Utility of clinical features for the diagnosis of seizures in the intensive care unit. $J$ Clin Neurophysiol. 2017;34(2):158161. [PubMed] doi:10.1097/ WNP.0000000000000335

15. Claassen J, Mayer SA, Kowalski RG, Emerson RG, Hirsch LJ. Detection of electrographic seizures with continuous EEG monitoring in critically ill patients. Neurology. 2004;62(10):17431748. [PubMed] doi:10.1212/01. WNL.0000125184.88797.62

16. Smith M, Meyfroidt G. Critical illness: the brain is always in the line of fire. 
Intensive Care Med. 2017;43(6):870873. [PubMed] [Free full text] doi:10.1007/s00134-017-4791-3

17. Rijsdijk, M., Leijten, F., \& Slooter AJ. Continuous EEG monitoring in the intensive care unit. Netherlands J Crit Care. 2008;12(4):157-162.

18. Ruiz AR, Vlachy J, Lee JW, Gilmore EJ, Ayer T, Haider $\mathrm{HA}$, et al. Association of periodic and rhythmic electroencephalographic patterns with seizures in critically ill patients. JAMA Neurol. 2017;74(2):181188. [PubMed] doi:10.1001/ jamaneurol.2016.4990

19. Al-Said YA, Baeesa SS, Shivji Z, Kayyali H, Alqadi K, Kadi G, et al. Non-convulsive seizures and electroencephalography findings as predictors of clinical outcomes at a tertiary intensive care unit in Saudi Arabia. Clin Neurol Neurosurg. 2018;171:95-99. [PubMed] doi:10.1016/j.clineuro.2018.06.002

20. Fogang $Y$, Legros B, Depondt C, Mavroudakis N, Gaspard N. Yield of repeated intermittent EEG forseizure detection in critically ill adults. Neurophysiol Clin. 2017;47(1):512. [PubMed] doi:10.1016/j. neucli.2016.09.001

21. Beghi E, Carpio A, Forsgren L, Hesdorffer DC, Malmgren $\mathrm{K}$, Sander JW, et al. Recommendation for a definition of acute symptomatic seizure. Epilepsia. 2010;51(4):671-675. [PubMed] [Free full text] doi:10.1111/j.15281167.2009.02285.x

22. Loho AM, Hakim M, Bestari AP, Budikayanti A, Kurniawan M, Indrawati $L A$, et al. Electroencephalography features and incidence non convulsive status epilepticus in metabolic encephalopathy at CIPTO Mangunkusumo National General Hospital. J Neurol Sci. 2017;381(2017):551. [PubMed] doi:10.1016/j.jns.2017.08.3760

23. Kaplan PW. The EEG in metabolic encephalopathy and coma. J Clin Neurophysiol. 2004;21(5):307318. [PubMed] doi: 10.1097/01. WNP.0000145004.22230.D5

24. Faigle R, Sutter R, Kaplan PW. The electroencephalography of encephalopathy in patients with endocrine and metabolic disorders. J Clin Neurophysiol. 2013;30(5):19. [Free full text] doi:10.1097/
WNP.0b013e3182a73db9

25. Holtkamp $M$, Meierkord $H$. Nonconvulsive status epilepticus: A diagnostic and therapeutic challenge in the intensive care setting. Ther $A d v$ Neurol Disord. 2011;4(3):169-181. [PubMed] [Free full text] doi:10.1177/1756285611403826

26. Nardone R, Brigo F, Trinka E. Acute symptomatic seizures caused by electrolyte disturbances. J Clin Neurol. 2016;12(1):21-33. [PubMed] [Free full text] doi:10.3988/ jcn.2016.12.1.21

27. Friedman D, Claassen J, Hirsch LJ. Continuous electroencephalogram monitoring in the intensive care unit. Anesth Analg. 2009;109(2):506523. [PubMed] doi:10.1213/ ane.0b013e3181a9d8b5

28. Claassen J, Taccone FS, Horn P, Holtkamp M, Stocchetti N, Oddo M. Recommendations on the use of EEG monitoring in critically ill patients: Consensus statement from the neurointensive care section of the ESICM. Intensive Care Med. 2013;39(8):1337-1351. [PubMed] doi:10.1007/s00134-013-2938-4

29. lanof JN, Anghinah $R$. Traumatic brain injury: An EEG point of view. Dement Neuropsychol. 2017;11(1):3-5. [PubMed] [Free full text] doi:10.1590/198057642016dn11-010002

30. Tatum WO. EEG interpretation: Common problems. Clin Pract. 2012;9(5):527-538. doi:10.2217/ cpr.12.51

31. Kim JA, Boyle EJ, Wu AC, Cole AJ, Staley KJ, Zafar S, et al. Epileptiform activity in traumatic brain injury predicts post-traumatic epilepsy. Ann Neurol. 2018;83(4):858-862. [PubMed] [Free full text] doi:10.1002/ ana.25211

32. Ronne-Engstrom E, Winkler T. Continuous EEG monitoring in patients with traumatic brain injury reveals a high incidence of epileptiform activity. Acta Neurol Scand. 2006;114(1):4753. [PubMed] doi:10.1111/j.16000404.2006.00652.x

33. Sinha SR, Hirsch LJ. Continuous EEG Monitoring in the intensive care unit. In: Ebersole JS, Nordli DR $\mathrm{HA}$, ed. Current Practice of Clinical Electroencephalography. Vol 53. 4th ed. Philadelphia: Wolters Kluwer Lippincott Williams \& Wilkins;
2014:543-597. CB09781107415324.004

34. Rots ML, van Putten MJAM, Hoedemaekers CWE, Horn J. Continuous EEG monitoring for early detection of delayed cerebral ischemia in subarachnoid hemorrhage: A pilot study. [PubMed] Neurocrit Care. 2016;24(2):207-216. doi:10.1007/ s12028-015-0205-y

35. Herman ST, Abend NS, Bleck TP, Chapman KE, Drislane FW, Emerson $R G$, et al. Consensus statement on continuous EEG in critically ill adults and children, part I: Indications. J Clin Neurophysiol. $\quad$ 2015;32(2):87-95. [PubMed] [Free full text] doi:10.1097/ WNP.0000000000000166

36. Jordan KG, Hirsch LJ. In nonconvulsive status epilepticus (NCSE), treat to burst-suppression: Pro and con. Epilepsia. 2006;47(SUPPL. 1):41-45. [PubMed] [Free full text] doi:10.1111/ j.1528-1167.2006.00659.x

37. McGrane S, Pandharipande PP. Sedation in the intensive care unit. Minerva Anestesiol. 2012;78(3):369380. [PubMed] [Free full text] doi:10.2147/CPAA.S26582

38. Friberg $\mathrm{H}$, Westhall $\mathrm{E}$, Rosén I, Rundgren $\mathrm{M}$, Nielsen N, Cronberg T. Clinical review: Continuous and simplified electroencephalography to monitor brain recovery after cardiac arrest. Crit Care. 2013;17(4):233. [PubMed] [Free full text] doi:10.1186/ cc12699

39. Hilkman DMW, Van Mook WNKA, Van Kranen-Mastenbroek VHJM. Continuous electroencephalographicmonitoring in the ICU: An overview of current strengths and future challenges. Curr Opin Anaesthesiol. 2017;30(2):192199. [PubMed] doi:10.1097/ AC0.0000000000000443

40. Eckhardt K, Kwok E. Electroencephalography in the intensive care unit: A technical assessment. C Proc. 2017;32.

41. Teplan M. Fundamentals of EEG measurement. Meas Sci Rev. 2002;2(2):1-11. doi:10.1021/ pr070350l

42. Usakli $A B$. Improvement of EEG signal acquisition: An electrical aspect for state of the Art of front end. Comput Intell Neurosci. 2010;2010. doi:10.1155/2010/630649

43. Singhal BS, Khadilkar S V. Neurology 
continuous electroencephalography

in the developing world. Handb

Clin Neurol. 2014;121:1773-1782.

[PubMed]
7020-4088-7.00114-0

44. Tan CT. Neurology in Asia. Neurology. 2015;84(6):623-
625. [PubMed] doi:10.1212/

WNL.0000000000001224

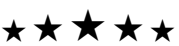

